Jacobs Heidi (Orcid ID: 0000-0001-7620-3822)

Buckley Rachel (Orcid ID: 0000-0002-5356-5537)

\title{
The impact of $A \beta$ and tau on prospective cognitive decline in older individuals
}

Reisa A. Sperling*, MD ${ }^{1,2}$, Elizabeth C. Mormino*, PhD ${ }^{1,3}$, Aaron P. Schultz, $\mathrm{PhD}^{1}$, Rebecca A. Betensky, $\mathrm{PhD}^{1,4}$, Kathryn V. Papp, $\mathrm{PhD}^{1,2}$, Rebecca E. Amariglio, $\mathrm{PhD}^{1,2}$, Bernard J. Hanseeuw, MD, PhD ${ }^{1,5}$, Rachel Buckley, $\mathrm{PhD}^{1,6}$, Jasmeer Chhatwal, MD, $\mathrm{PhD}^{1}$, Trey Hedden, $\mathrm{PhD}{ }^{1,5}$, Gad A. Marshall, $\mathrm{MD}^{1,2}$, Yakeel T. Quiroz, $\mathrm{PhD}^{1,7}$, Nancy J. Donovan, $\mathrm{MD}^{1,2,7}$, Jonathan Jackson, $\mathrm{PhD}^{1}$, Jennifer R. Gatchel, $\mathrm{MD}, \mathrm{PhD}^{1,7,8}$, Jennifer $\mathrm{S}$. Rabin, $\mathrm{PhD}^{1,7}$, Heidi Jacobs, $\mathrm{PhD}^{1}$, Hyun-Sik Yang, $\mathrm{MD}^{1,2}$, Michael Properzi, $\mathrm{BS}^{1}$, Dylan R. Kirn, $\mathrm{MPH}^{1}$, Dorene M. Rentz, PsyD ${ }^{1,2}$, Keith A. Johnson*, MD ${ }^{1,2,5,9}$

${ }^{1}$ Harvard Aging Brain Study, Department of Neurology, Massachusetts General Hospital, Harvard Medical School, Boston, Massachusetts, USA

${ }^{2}$ Center for Alzheimer Research and Treatment, Department of Neurology, Brigham and Women’s Hospital, Harvard Medical School, Boston, Massachusetts

${ }^{3}$ Department of Neurology, Stanford Medical School, Palo Alto, California

${ }^{4}$ Department of Biostatistics, Harvard T.H. Chan School of Public Health, Boston, Massachusetts, USA

${ }^{5}$ Department of Radiology, Massachusetts General Hospital, Harvard Medical School, Boston

${ }^{6}$ Florey Institute, University of Melbourne, Parkville, Victoria, Australia

${ }^{7}$ Department of Psychiatry, Massachusetts General Hospital, Harvard Medical School, Boston, Massachusetts, USA

${ }^{8}$ Division of Geriatric Psychiatry, McLean Hospital, Harvard Medical School, Belmont, Massachusetts, USA

${ }^{9}$ Division of Molecular Imaging, Massachusetts General Hospital, Harvard Medical School, Boston

This is the author manuscript accepted for publication and has undergone full peer review but has not been through the copyediting, typesetting, pagination and proofreading process, which may lead to differences between this version and the Version of Record. Please cite this article as doi: 10.1002/ana.25395

This article is protected by copyright. All rights reserved. 
*These authors contributed equally to the work in this study.

Corresponding Author: Reisa Sperling, MD, Reisa Sperling, M.D.

Center for Alzheimer Research and Treatment, Harvard Aging Brain Study Brigham and Women's Hospital and Massachusetts General Hospital 60 Fenwood Avenue Boston, MA 02115

617-525-8675

reisa@rics.bwh.harvard.edu

Running head: Amyloid, Tau, and Cognition in Normal Elderly

Number of words in abstract: 249

Number of words in full manuscript: 4815

Number of Figures: 4

Number of Tables: 3

Number of Supplementary Tables: 1 


\begin{abstract}
:
Objectives: Amyloid-beta $(A \beta)$ and tau pathologies are commonly observed among clinically normal older individuals at post-mortem and can now be detected with in vivo neuroimaging. The association and interaction of these proteinopathies with prospective cognitive decline in normal aging and preclinical Alzheimer's disease (AD) remains to be fully elucidated.
\end{abstract}

Methods: One hundred and thirty-seven older individuals (age=76.3 \pm 6.22 years)

participating in the Harvard Aging Brain Study underwent $A \beta\left({ }^{11} \mathrm{C}\right.$-Pittsburgh Compound B) and tau ( ${ }^{18}$ F-Flortaucipir) positron emission tomography (PET) with prospective neuropsychological assessments following PET imaging (mean number of cognitive visits $=2.8 \pm 1.1)$. Tau and $\mathrm{A} \beta \mathrm{PET}$ measures were assessed in regions of interest $(\mathrm{ROI})$ as well as vertex-wise map analyses. Cognitive change was evaluated with Memory and Executive Function composites. 
Results: Higher levels of $A \beta$ and tau were both associated with greater memory decline, but not with change in executive function. Higher cortical $A \beta$ was associated with higher tau levels in all ROI, independent of age, and very elevated levels of tau were observed primarily in $\mathrm{CN}$ with elevated $\mathrm{A} \beta$. A significant interaction between tau and $\mathrm{A} \beta$ was observed in both ROI and map level analyses, such that rapid prospective memory decline was observed in participants who had high levels of both pathologies.

Interpretation: Our results are consistent with the supposition that both $A \beta$ and tau are necessary for memory decline in the preclinical stages of AD. These findings may be relevant for disambiguating aging and early cognitive manifestations of $\mathrm{AD}$, and to inform secondary prevention trials in preclinical AD. 


\section{Introduction}

The pathophysiologic process of Alzheimer's disease (AD) is thought to commence more than a decade before the stage of dementia. ${ }^{1-3}$ The two hallmark pathologies of $\mathrm{AD}$, amyloid- $\beta(\mathrm{A} \beta)$ and tau aggregates, are commonly observed at postmortem in clinically normal older individuals $(\mathrm{CN})$ and can now be detected in vivo with PET imaging techniques. ${ }^{4-7}$ In this study, we investigated the association of amyloid- $\beta(A \beta)$ and tau imaging markers with prospective cognitive trajectories in $\mathrm{CN}$ participating in the Harvard Aging Brain Study (HABS).

The late-life accumulation of these proteins may play a critical role in cognitive decline, and several secondary prevention trials are currently evaluating the efficacy of anti-A $\beta$ interventions to slow tau accumulation and prevent cognitive decline in preclinical stages of AD. First, we sought to understand the relationships among age, in vivo measures of $A \beta$ and regional tau pathology in CN. Specifically, we evaluate recently acquired PET data to assess the relationship of age and $\mathrm{A} \beta$ to tau pathology in the medial temporal lobe and neocortical regions. We further aimed to evaluate the association of $A \beta$ and tau with prospective cognitive change, focusing on memory and executive function (EF) as these domains have been widely reported to decline in both aging and very early AD. Multiple studies have reported that $\mathrm{CN}$ with elevated $\mathrm{A} \beta$ demonstrate faster rates of cognitive decline in both memory and executive function domains, ${ }^{8-12}$ but there are very limited reports to date evaluating the association of both $A \beta$ and tau with longitudinal 
trajectories of cognitive performance in $\mathrm{CN}^{7,13,14}$ and these studies have primarily examined cross-sectional or retrospective cognitive data.

Several related questions motivated our research. It has been posited that $A \beta$ may be necessary but not sufficient to predict future cognitive decline in the preclinical stages of AD. ${ }^{15}$ Previous studies have suggested that $\mathrm{CN}$ with abnormalities in both $\mathrm{A} \beta$ and tau measures in cerebrospinal fluid (CSF) are more likely to demonstrate cognitive decline over long-term follow-up. ${ }^{16,17}$ One potential advantage of tau PET over CSF is the ability to investigate the relevance of specific anatomic regional tau deposition. Autopsy reports suggest that tau deposition in the entorhinal cortex (EC) is nearly ubiquitous in advanced aging, whereas spread into the surrounding temporal neocortex is less commonly observed among clinically normal older individuals, particularly in the absence of substantial $A \beta$ pathology. ${ }^{18}$ It remains unclear whether EC tau accumulation is relatively "benign” and associated with “normal aging”, such that neocortical tau is required for cognitive decline. Although both memory and executive function decline have been associated with elevated amyloid, the relative specificity of decline in these cognitive domains related to tau has not yet been examined. Moreover, it is unknown whether similar patterns of tau associated cognitive change are observed among CN with low levels of cortical amyloid (A $\beta-)$ compared to those with elevated levels of amyloid $(A \beta+)$. Finally, we modeled multiple factors that may influence longitudinal cognitive performance, including age, APOE, sex, and education, to investigate the relative 
contributions of $A \beta$, regional tau, and the potential synergy of these pathologies in the prediction of prospective cognitive decline.

\section{Methods \\ Participants}

One hundred and thirty-seven participants from HABS were included in this study. HABS participants were recruited from the community through media and outreach events, and screened for major neurologic, psychiatric or unstable medical illnesses. At study entry, all HABS participants were clinically normal (CN), had a global Clinical Dementia Rating $(\mathrm{CDR})=0$, Mini Mental Status Examination $(\mathrm{MMSE}) \geq 27$ with educational adjustment, and performed within education-adjusted norms on Logical Memory Delayed Recall.

All participants provided written informed consent prior to undergoing any study procedures. The HABS protocol is approved annually by the Partners Human Research Committee.

Neuropsychological evaluations 
Participants are evaluated annually with a battery of cognitive and functional assessments, including tests of episodic memory, executive function, global cognition, and the CDR. For this study, we combined several neuropsychological tests to create two composites, covering memory and executive function, to estimate change across several tests within each domain. Given the mid-entrance of tau PET into HABS, we focused on tests administered annually (as opposed to those administered only on selected years) to ensure that consistent composites were used across participants and time points. Individual tests were z-transformed using the baseline sample and averaged to form the composites. $^{19}$

Episodic memory composite: The episodic memory composite included three measures: The Free and Cued Selective Reminding Test (FCSRT) is a multi-modal associative memory measure. Two primary FSCRT scores were included in the composite: 1) Free Recall is the sum of items freely recalled (up to a total of 48) and 2) Total Recall is the sum of free and cued recall (up to a total of 48). The 6-Trial Selective Reminding Test (SRT) is comprised of 12 words, learned over 6 trials. 3) Delayed Recall on the SRT assessed after a 10-minute delay was included in the composite.

Executive Function Composite: The executive function composite included three measures: 1) The Digit-Symbol Substitution test from the Wechsler Adult Intelligence Scale - Revised measures the ability to rapidly transcribe a geometric symbol to the correct corresponding number within 90 seconds. 2) Trails B measures the speed to 
accurately connect letters and numbers in alternating sequence. Trails B is logtransformed and inverted so that higher values reflect better performance. 3) Phonemic Letter Fluency measures the number of words generated to three letters (F-A-S), each over 60 seconds.

\section{Molecular Imaging}

$\mathrm{C}^{11}$-Pittsburgh Compound B (PiB) and $\mathrm{F}^{18}$-Flortaucipir (FTP) (previously known as AV-1451 or T807) PET tracers were synthesized and administered at Massachusetts General Hospital (Siemens ECAT EXACT HR+ scanner). For both PiB and FTP-PET cerebellar grey matter was used as the reference region from the Freesurfer atlas as previously described. ${ }^{7}$ For PiB, distribution volume ratio (DVR) images were created with Logan plotting (40-60min), and PiB values were extracted from a global cortical aggregate (given the very high degree of collinearity among neocortical regions) and normalized to cerebellar grey. $A \beta$ was treated as a continuous variable in statistical models. Participants were dichotomized into $A \beta$ - and $A \beta+$ groups using a DVR cut off of $1.20,{ }^{20}$ for additional analyses and visualization purposes.

FTP (tau) PET imaging was introduced mid-study into HABS as this technology was not available at study initiation. We utilized the first FTP scan acquired in HABS for each participant, and the corresponding PiB amyloid scan acquired closest in time (years between PiB and FTP, $M=0.32, S D=0.44$ ). FTP scans were acquired at Year 4 of HABS 
or later for most participants, but a minority of participants who enrolled more recently in HABS underwent FTP at their earlier HABS visits (Table 1).

FTP-PET measures were computed as standardized uptake value ratios (SUVRs) in three individually defined anatomic regions of interest (using FreeSurfer 6.0): entorhinal cortex (EC), hippocampus (Hip), and inferior temporal (IT) cortex, which commonly exhibit early Tau pathology among CN. ${ }^{7,21-23}$ Because of reports of off-target binding in choroid plexus adjacent to the hippocampus, we residualized the Hip FTP values for mean choroid plexus signal. ${ }^{24}$

\section{Statistical Analyses:}

Cross-sectional relationships between age, $\mathrm{A} \beta(\mathrm{PiB})$, and tau (FTP) were examined with linear regression models. When predicting tau, we explored the independent effects of $A \beta$ and age, as well as the interaction between $A \beta$ and age.

To visually examine the associations between cognitive change as a function of both regional tau and $A \beta$, cognitive slopes for both memory and executive function were extracted from a simple linear mixed model with the cognitive variable as the outcome and a time term as the only predictor (by extracting the random slope term from this model for each participant). These slopes were plotted against regional tau, with individual data points additionally coded by $\mathrm{A} \beta$ group. Before plotting, cognitive slopes and regional tau were residualized by age, sex, education, and time of tau scan. These 
plots enabled visualization of the overall pattern between these variables, whereas all associations were tested formally in linear mixed models (LMM, Supplementary Table 1).

LMM were conducted to examine prospective change in cognition following most recent tau and A $\beta$ PET imaging, separately for memory and executive function composite scores. The time term in these models was relative to the neuropsychological testing session within one year of the date of the tau PET scan. The mean prospective follow up period was 2.04 years, with a mean number of cognitive visits $=2.8 \pm 1.1$ ). Of the 137 participants, 12 had a single neuropsychological testing visit, 31 had two visits, 53 had three visits, and 41 had four visits. Thus, the majority of individuals (69\%) had 3 or more visits. Although the 12 participants that had a single time point did not contribute directly to the model estimates of cognitive change over time in our LMMs, these data were included since they contribute to the estimation of the model main effects.

The same time term was used in models examining change related to A $\beta$ PET imaging, since A $\beta$ PET imaging for each participant was selected to be close in time to the tau scans All models included age at the tau scan, time (relative to the neuropsychological testing session closest to the tau PET scan), APOE status ( $\varepsilon 4$ carrier versus non-carrier), education, sex, time of tau scan relative to time of entry into the HABS Study (to account for the variance in when tau PET was first acquired across participants), as well as the interaction between time and all covariates (time $\mathrm{x}$ age, time x APOE, etc). Random intercepts were included in all models. 
We first examined $A \beta$ and regional tau as predictors of cognitive change in separate LMMs, by examining the interaction of each PET variable with the time term (Supplemental Table 1). We subsequently modeled $\mathrm{A} \beta$ and tau as simultaneous predictors within the same model (with the same covariates listed above). Then we added the threeway interaction between $A \beta$, tau and time to this model to determine whether the association between tau and cognitive decline was modified by $\mathrm{A} \beta$ levels $(\mathrm{A} \beta \mathrm{x}$ Regional Tau x Time).

Exploratory, vertex-wise whole brain analyses to investigate associations across all brain regions were performed with GLM_Flex_Fast (싸rtools.mgh.harvard.edu). Models were run examining the relationship between 1) PiB-PET burden and 2) FTP-PET with change on the Memory and EF composites (extracted from linear mixed models). Additionally, we explored vertex wise map analyses of PiB-PET interacting with tau R0I and FTP-PET vertex wise map interacting with A $\beta$ ROI to assess the anatomic patterns of A $\beta$ by tau interactions associated with memory decline. All analyses controlled for age and all maps are displayed at a $\mathrm{t}$-value threshold of $\mathrm{t}= \pm 3.362$, or $\mathrm{p}<0.001$.

All p values were 2-sided. We did not correct for multiple comparisons for the ROI analyses, as we examined only ROI of independent a priori interest, and used composite neuropsychological measures. However, we did require a stringent statistical threshold ( $<<0.001$, as above) for the whole brain voxel wise analyses to account for multiple comparisons. 
We also repeated the primary analyses using partial volume correction (PVC) using the geometric transform matrix method, as implemented in Freesurfer $6.0,{ }^{25}$ assuming a uniform $6 \mathrm{~mm}$ point spread function. Utilization of PVC values did not alter the overall pattern of results.

\section{Results}

Demographics at the time of PET scanning are presented for the entire group and by dichotomized A $\beta$ groups (Table 1). As expected from other reports, $\mathrm{CN}$ who were classified as $A \beta+$ were slightly older $(\mathrm{p}=0.015)$ and more likely to be $A P O E \varepsilon 4$ carriers $\left(\chi^{2}=31.9, \mathrm{p}<0.001\right)$. At the time of tau scanning, logical memory scores were lower in the $\mathrm{A} \beta+$ group ( $\mathrm{p}=0.013)$. All participants were clinically normal (Global CDR=0) at entry into HABS. Three participants progressed to a diagnosis of Mild Cognitive Impairment (MCI) as determined by diagnostic consensus conference, within one year of their tau PET imaging (all three participants who transitioned to MCI were $A \beta+$ ). We repeated the analyses excluding these three participants, which did not alter any of the results presented below.

Age and $\mathrm{A} \beta$ Influence Degree of Tau Pathology

We first investigated the relationships of age (at time of PET scan), A $\beta$ and tau using linear regression models. Older age was associated with higher continuous cortical $\mathrm{A} \beta$ (beta: $0.0067 \pm 0.0031, \mathrm{r}=0.18, \mathrm{p}=0.034$ ). Older age was also significantly associated with elevated tau in EC (beta: 0.0044 $\pm 0.0017, \mathrm{r}=0.22, \mathrm{p}=0.009$ ) and IT (beta: 
$0.0042 \pm 0.0012, r=0.28, p<0.001$ ), but did not reach statistical significance for Hip (beta:

$0.0024 \pm 0.0012, \mathrm{r}=0.16, \mathrm{p}=0.054)$.

We then examined the relationship between continuous $A \beta$ levels and tau, controlling for age. Higher $A \beta$ was associated with higher tau, independent of age, in all tau ROI (EC beta: $0.2458 \pm 0.0410, \mathrm{r}_{\mathrm{p}}=0.46, \mathrm{p}<0.001$; Hip beta: $0.1329 \pm 0.0320, \mathrm{r}_{\mathrm{p}}=0.34$, $\mathrm{p}<0.001$; and IT beta: $0.1675 \pm 0.0306, \mathrm{r}_{\mathrm{p}}=0.43, \mathrm{p}<0.001$ ). The interaction between age and continuous $A \beta$ was not significant for any Tau ROI (p-values>0.34). Across all participants, the three tau ROI showed significant correlations with each other (EC Tau versus Hip: $r=0.626$, EC versus IT: $r=0.698$, Hip versus IT: $r=0.607$; $p$-values $<0.0001$ ). An exploratory whole brain analysis, covarying PiB DVR into the vertex-wise FTP PET map, controlling for age, also demonstrated that higher $\mathrm{A} \beta$ was associated with higher tau in temporal regions bilaterally, with strongest correlations observed in the right medial temporal lobe (Figure 1).

Amyloid and tau are each associated with longitudinal memory decline

Visualization of cognitive change as a function of regional tau and $A \beta$ group revealed a consistent pattern across ROI with respect to memory, such that $\mathrm{A} \beta+$ individuals with greater tau showed the greatest memory decline (Figure 2A-C). Regional tau was not associated with memory change among $A \beta$ - individuals, although it should be noted that $A \beta$ - participants did not show very high levels of tau in these regions. 
Furthermore, regional tau was not associated with change in EF in either $A \beta+$ or $A \beta-$ groups (Figure 2D-F).

We formally tested these associations between regional tau, $A \beta$, and cognitive change in a series of linear mixed models. We first investigated the association of prospective change in cognitive performance with $A \beta$ and tau separately, controlling for age, education, APOE4, sex, and the time of tau scan. Continuous levels of A $\beta$ were associated with greater longitudinal decline in the memory composite (beta: $-0.641 \pm 0.163, \mathrm{p}<0.001$ ), whereas age was not related to change in memory $(-0.0053 \pm 0.0053, p=0.32)$. Interestingly, age was significantly related to the $\mathrm{EF}$ composite (beta: $-0.009 \pm 0.003, \mathrm{p}<0.001$ ), and we observed only a weak relationship between change in $\mathrm{EF}$ and continuous mean cortical $\mathrm{A} \beta$ (beta: $-0.146 \pm 0.079, \mathrm{p}=0.067$ ). The pattern of results was very similar when $A \beta$ was treated dichotomously rather than continuously.

Greater tau in all three anatomic ROI was related to greater decline in the memory composite (EC: $-0.594 \pm 0.269$, $\mathrm{p}=0.028$; Hip: $-1.117 \pm 0.393$, $\mathrm{p}=0.005$; IT: $-0.889 \pm 0.369$, $\mathrm{p}=0.017)$. In contrast, the relationships between tau and EF did not reach statistical significance and were considerably smaller in magnitude than those observed for memory, as evident by the model beta estimates for EF that are approximately 4 times smaller (EC: $-0.144 \pm 0.130 \mathrm{p}=0.28$; Hip: $0.025 \pm 0.195$, $\mathrm{p}=0.90$; IT: $-0.201 \pm 0.184, \mathrm{p}=0.27$ ) than the LMMs predicting memory shown above. 
To evaluate relationships of amyloid and tau with memory or EF across a more extensive range of anatomy, we also examined the association of longitudinal cognitive slopes using whole brain map vertex-wise analyses of the PiB and FTP SUVr images. Higher levels of $\mathrm{A} \beta(\mathrm{PiB})$ in medial orbital and lateral prefrontal, posterior cingulate, precuneus, and lateral parietal regions were associated with worsening memory performance ( $<<0.001$; Figure 3A). We note that the regions demonstrating this significant relationship nearly completely overlaps the set of regions in our cortical aggregate ROI and strongly resembles the Stage II of amyloidosis, described by Grothe and colleagues. ${ }^{26}$ We found increasing tau (FTP) to be associated with longitudinal memory change in medial and inferior temporal regions (Figure 3B). The regions involved were spatially aligned with the FTP map that showed an association with increasing levels of PiB (as in Figure 1). Interestingly, the map level analyses suggested a stronger relationship between tau in right medial temporal regions with mean cortical $A \beta$, but demonstrated a particularly strong relationship between tau in the left medial temporal lobe and decline on the memory composite, perhaps because our memory composite is weighted towards verbal memory measures. Similar to ROI analysis results, we did not find evidence of a significant relationship between EF change and FTP in any region in the vertexwise analyses, using a similar threshold of $\mathrm{p}<0.001$ (data not shown).

Amyloid and tau interact to influence cognitive decline 
We then investigated the independent and synergistic effects of continuous values of $\mathrm{A} \beta$ and tau on memory and EF change, using both PiB and FTP values as simultaneous predictors (separately for each of the three FTP regions). In all models predicting memory change, the 3-way interaction terms between $\mathrm{A} \beta$, tau in all ROI and time were significant (Table 2). Examination of EF models revealed that the only significant independent predictor of prospective change in EF across all tau regions was Age x Time (all ROI $\mathrm{p}<0.002$ ). No other term (APOE, sex, education or time of tau scan) was significantly associated with change in memory or EF, once age and the 3-way interaction of $\mathrm{A} \beta$ by tau ROI by time were included in the models.

We also explored the interaction of $A \beta$ and tau using whole-brain, vertex-wise PiB and FTP maps. The interaction of each of the tau ROI into the PiB map to predict longitudinal memory change revealed a similar pattern of to our aggregate A $\beta$ ROI. Large areas of precuneus, lateral prefrontal (but limited orbital prefrontal), and lateral parietal cortex demonstrated significant interactions with inferior temporal tau ROI (Figure 3C), suggesting that multiple cortical regions with $\mathrm{A} \beta$ deposition contribute to the interaction with tau in predicting memory decline. We did not observe any regions on the PiB map that interacted with tau to predict change in EF. Conversely, the tau vertex-wise map interacting with the cortical aggregate $\mathrm{A} \beta$ ROI to predict memory change (Figure 3D) revealed medial and lateral temporal tau regions predicting memory decline. Interestingly, the FTP map with A $\beta$ interaction revealed a similar pattern to the FTP map 
alone (Figure 3B) but showed even stronger associations with memory change, again suggesting that higher tau in the setting of higher $A \beta$ is associated with the greatest prospective memory decline.

Given the significance of the 3-way interaction in the memory models, the relationship of $A \beta$, tau and memory was therefore interpreted in the context of this interaction in all subsequent models. These models revealed consistent evidence for a synergistic association such that individuals with both elevated $A \beta$ and tau levels showed the greatest memory decline. Figure 4 highlights that a negative relationship between A $\beta$ burden as a continuous measure and memory change emerges around values of 1.1 SUVR in EC Tau. Likewise, the negative effect of EC Tau on memory emerges at around PiB DVR values of 1.4. This multiplicative effect of $A \beta$ and tau was consistent across all examined tau regions, as indicated by the significant interaction term across all three models.

Finally, to further explore the interaction between $A \beta$ and tau with memory decline, models were repeated treating amyloid as a dichotomous variable to estimate the effect of tau on memory change in $A \beta$ - and $A \beta+$ groups (Table 3 ). These models provide further evidence that greater tau was significantly associated with greater decline in memory, specifically within the $A \beta+$ group, consistent with the visualization in Figure 2. We did not observe evidence of tau-related memory decline within the A $\beta$ - group. In fact, the directionality of the relationship between EC tau and memory change among the 


\begin{abstract}
A $\beta$ - was paradoxically positive, an effect that is likely spurious and driven by the more restricted range of EC tau values present in the $\mathrm{A} \beta$ - group (mean EC tau $=1.084 \pm 0.087$; range $=0.933-1.298$ ) compared to the $\mathrm{A} \beta+$ group (mean EC tau=1.201 \pm 0.157 ; range $=0.962-1.495)$.
\end{abstract}

\title{
Discussion:
}

This study assessed the association of $A \beta$ and tau pathology, as estimated with in vivo imaging markers, with prospective longitudinal cognitive trajectories among a group of well-characterized CN. Our findings suggest that the hallmark pathologies of AD are synergistically associated with longitudinal memory decline. Specifically, the fastest rates of memory decline were observed in $\mathrm{CN}$ with higher levels of both $\mathrm{A} \beta$ and tau on PET imaging.

Our findings using neuroimaging markers are consistent with previous reports using CSF, suggesting that greater cognitive decline is observed in CN with abnormalities of both CSF A $\beta$ and phospho-tau. ${ }^{16,17,27-29}$ These findings are also consistent with laboratory work suggesting that tau is likely the primary mechanism of A $\beta$-related neurotoxicity, ${ }^{30}$ and previous work using other imaging markers of neurodegeneration, ${ }^{31-34}$ suggesting that $\mathrm{A} \beta$ pathology in isolation may be insufficient to drive imminent cognitive decline. 
Our findings may also be relevant to the question as to whether tau, in the setting of low $A \beta$, might underlie age-related decline in episodic memory, given the nearly ubiquitous presence of tau pathology in the EC (Braak Stage 1-2) by age $80,{ }^{18}$ and the recent interest in "primary age-related tauopathy" (PART), ${ }^{35}$ defined as early neocortical tau accumulation with absent or low $\mathrm{A} \beta$ pathology (Thal phase $<3$ corresponding approximately to PET A $\beta$-) in very elderly individuals (average ages 88-90). These PART studies have reported somewhat variable findings regarding the relationship to cognition, with one recent study finding no association between episodic memory measures and tau pathology, despite substantial hippocampal atrophy, in the setting of low $\mathrm{A} \beta$ pathology. ${ }^{36}$ There have also been two reports of memory decline, using primarily retrospective cognitive trajectories, associated with elevated tau PET measures among $A \beta$ - participants from the Berkeley Aging Cohort Study. ${ }^{13,14}$ Our overall results are consistent with the Berkeley group findings, however, we did not find supporting evidence for tau-associated cognitive decline among $A \beta$ - in any region or in any cognitive domain. Our cohort included a somewhat larger number of participants, who were slightly younger overall, and were evaluated prospectively using somewhat different cognitive testing instruments. Furthermore, our $A \beta$ - group did not show very elevated levels of tau. Our finding that memory decline requires the synergistic relationship of both elevated $\mathrm{A} \beta$ and tau was highly consistent across all analyses (i.e., 
using continuous $A \beta$, dichotomous $A \beta$ groups, with or without partial volume correction in the tau ROI, and in whole-brain vertex-wise map analyses).

We replicated previous reports that $\mathrm{A} \beta$ and tau PET measures are correlated, even among $\mathrm{CN} .{ }^{1,7,37,38}$ Furthermore, we show that it is only a subset of $A \beta+$, with higher levels of tau detected by PET imaging, who demonstrate prospective memory decline. Our results are consistent with the hypothetical model that accumulating $A \beta$ may be associated with accelerated tau spreading, which in turn underlies cognitive decline in preclinical AD. ${ }^{39}$ However, more direct evidence for the temporal ordering in this model will require evaluation of simultaneous change measures of both $\mathrm{A} \beta$ and tau biomarkers.

We wanted to ascertain whether tau PET signal in three specific anatomic regions that often demonstrate tau pathology in $\mathrm{CN}$ at postmortem ${ }^{1,18}$ exhibit differential relationships with $A \beta$ and cognitive decline. While tau PET measures are generally consistent with the Braak staging scheme ${ }^{40}$ they are not necessarily equivalent to tangle counts (the basis of Braak staging), but rather correspond to the wider range of PET binding site substrates on tau pathology, including neuropil threads and the coronae of neuritic amyloid plaques observed in recent autoradiographic studies. ${ }^{41}$ We measured tau signal in the EC, an early site of allocortical tau deposition; ${ }^{18}$ the hippocampus because of its critical role in episodic memory function and early involvement presumably due to tau spread from EC, ${ }^{42}$ and IT as a proxy region for early neocortical tau spreading., ${ }^{1,13,23,43}$ We then investigated whether there were other regions that demonstrated similar 
relationships with $A \beta$ or cognitive decline using exploratory whole brain analyses, but found that our individual ROI well represented the areas showing significant tau associations.

Notably, we did not find differences among tau regions in the associations with age, $A \beta$, or memory decline. Indeed, EC tau, sometimes thought of as primarily agerelated and more "benign”, actually showed the strongest correlation with A $\beta$. Furthermore, all three regions, including the EC, demonstrated tau-related memory decline, that was only evident in the $A \beta+$ group, perhaps due to the finding that $A \beta$ showed lower levels of tau in all regions. Our results are consistent with early autopsy work by Price, Morris, and colleagues, highlighting evidence that elevated EC tau in CN, associated with $\mathrm{A} \beta$ plaques, represents preclinical AD. ${ }^{1}$ Given the co-linearity among these regions wherein participants with high EC tau also demonstrate higher levels of hippocampal and neocortical tau, it is difficult to ascertain whether tau spreading beyond the $\mathrm{EC}$ is required for memory decline among $\mathrm{A} \beta+\mathrm{CN}$. Using map-level analyses, we also found evidence that multiple cortical areas with $\mathrm{A} \beta$ deposition contributed to the interaction with tau associated with memory decline. Future studies with serial tau PET imaging in addition to longitudinal cognitive testing will further elucidate this question.

Interestingly, we did not observe a strong relationship between either $A \beta$ or tau with EF, despite previous evidence of decline in EF associated with elevated $\mathrm{A} \beta$ in a larger sample from $\mathrm{HABS}^{10}$ and other reports in the literature. ${ }^{8,9,12}$ Thus, we may be 
underpowered to detect change with the smaller sample size with both $A \beta$ and tau imaging using this EF composite. However, the stronger and consistent relationship between both $A \beta$ and tau and the memory composite is consonant with the hypothesis that episodic memory may be particularly vulnerable to early AD pathology. ${ }^{44}$

\section{Limitations:}

Tau FTP PET imaging was introduced mid-study to HABS, and thus we are able to investigate only relatively short trajectories of prospective longitudinal change. Because preliminary reports with longitudinal FTP PET suggest that tau accumulation may be much more dynamic than $A \beta$, we chose to investigate only prospective cognitive trajectories, as we did not have tau PET available at HABS baseline. Although over twothirds of our participants had at least three cognitive testing visits, 31 percent of the participants in our analysis had only one or two neuropsychological testing visits, which may limit our ability to reliably measure longitudinal change. Nevertheless, we did find significant synergistic relationships between $\mathrm{A} \beta$ and tau predicting memory decline over this short time period, suggesting $\mathrm{CN}$ with elevated levels of both pathologies are at very high risk for imminent decline. Longitudinal studies with serial A $\beta$ and tau PET are ongoing to investigate the relative rates of accumulation of these pathologies and the temporal proximity to cognitive decline.

p. 23

This article is protected by copyright. All rights reserved. 
It is important to acknowledge that FTP (AV-1451) and tau PET tracers in general, are relatively new, with reports of off-target binding with multiple tau tracers. ${ }^{41}$ Most of the regions with reported off-target binding are outside the ROI investigated in this study. However, the choroid plexus, which is adjacent to the hippocampus, may be particularly susceptible to off-target FTP binding and thus we performed analyses residualizing the hippocampal signal using an anatomically defined choroid plexus region. $^{24}$

Although we recruited the HABS cohort across strata based on age and socioeconomic status, the majority of HABS participants have at least some advanced education, are overall healthy, and may have substantial cognitive reserve. ${ }^{45}$ The neuropsychological tests utilized in HABS were chosen to be sensitive to decline associated with both "normal aging” and the earliest stages of AD. It is possible, however, that other neuropsychological measures would demonstrate evidence of decline in the setting of relatively low levels of $A \beta$ or tau accumulation, or across a larger age range. Finally, it is notable, that even with age, education, gender, $\mathrm{APOE}, \mathrm{A} \beta$, tau, and their interactions in the models, we are still only explaining a fraction of the variance in the short-term trajectories of memory or EF change. Thus, there are likely other contributing factors, including cerebrovascular disease, accumulation of TDP-43, loss of dopaminergic projections, or the synergistic effects of these processes with early AD pathology, that are not fully captured with the current molecular imaging markers. 


\section{Implications:}

Our findings provide further evidence to support the hypothesis that both of the hallmark pathologies of $\mathrm{AD}$, i.e. $\mathrm{A} \beta$ and tau, are required for rapid cognitive decline during the preclinical stages of AD. Thus, selecting individuals with evidence of higher levels of both pathologies might provide greater power in clinical trials to detect a therapeutic effect on rate of cognitive decline, particularly over a relatively short timeframe. ${ }^{17,46}$ However, it is possible that anti-A $\beta$ monotherapy will have more limited efficacy once substantial tau pathology is present, especially if there is an uncoupling of these pathophysiological processes as the disease progresses. ${ }^{47}$

Given the association between $A \beta$ and tau, and the observation that elevated levels of neocortical tau are primarily observed in the high $\mathrm{A} \beta$ group, it is possible that decreasing $A \beta$ accumulation might slow memory decline through preventing further accumulation of tau. This hypothesis is being tested in several ongoing secondary prevention trials in both biomarker-at-risk ${ }^{48}$ and in genetic-at-risk cohorts. ${ }^{49,50}$ Our findings do suggest the possibility, however, that once $A \beta$ and tau have begun to accumulate, particularly if there is already subtle memory decline apparent, in the later stages of preclinical $\mathrm{AD}$, combination therapies may be needed to maximally impact the clinical course of the disease. 
Acknowledgments: The Harvard Aging Brain Study is funded by the National Institute on Aging (P01AG036694) with additional support from several philanthropic organizations. Several co-authors’ contributions were also supported by career development awards: ECM and PV are supported by K01 awards from NIA; REA, JC and KVP are supported by K23 awards from NIA; JSR is supported by a CIHR postdoctoral fellowship award; JRG is supported by the BrightFocus Foundation and the Alzheimer's Association; KVP, HSJ, and REA are also supported by the Alzheimer's Association; HILJ is supported by a Marie Sklodowska-Curie Global Fellowship within the European Union's Horizon 2020 Research and Innovation Programme; YTQ is supported by the NIH Common Fund and JDJ was supported by an Administrative Supplement to P01AG036694.

We also wish to thank the research staff and the very dedicated participants of the Harvard Aging Brain Study.

\section{Author Contributions}

Conception and design of the study: R.A.S., E.C.M., R.A.B., K.A.J.; Acquisition and analysis of data, interpretation of results, and editing of manuscript: R.A.S., E.C.M., A.P.S., R.A.B., K.V.P, R.E.A., B.J.H., R.B., J.C., T.H., G.A.M., Y.T.Q., N.J.D., J.J., 
J.R.G., J.S.R., H.J., H.Y., M.P., K.R.K., D.M.R., K.A.J.; Drafting the text, preparing the figures, and final preparation of the manuscript: R.A.S., E.C.M., A.P.S., R.A.B., K.A.J.

\section{Potential Conflicts of Interest}

Drs. Sperling and Johnson are involved in public-private partnership clinical trials sponsored by NIH and Eli Lilly and Co. who owns the distribution rights to Flortaucipir (AV-1451), but they do not have any personal financial relationship with Eli Lilly. 


\section{References}

1. Price JL, Morris JC. Tangles and plaques in nondemented aging and "preclinical" Alzheimer's disease. Ann Neurol. 1999;45(3):358-68.

2. Rowe CC, Bourgeat P, Ellis KA, et al. Predicting Alzheimer disease with betaamyloid imaging: results from the Australian imaging, biomarkers, and lifestyle study of ageing. Ann Neurol. 2013 Dec;74(6):905-13.

3. Jack CR, Jr., Knopman DS, Jagust WJ, et al. Tracking pathophysiological

processes in Alzheimer's disease: an updated hypothetical model of dynamic biomarkers. Lancet Neurol. 2013 Feb;12(2):207-16.

4. Klunk WE, Engler H, Nordberg A, et al. Imaging brain amyloid in Alzheimer's disease with Pittsburgh Compound-B. Ann Neurol. 2004 Mar;55(3):306-19.

5. Chien DT, Bahri S, Szardenings AK, et al. Early clinical PET imaging results with the novel PHF-tau radioligand [F-18]-T807. J Alzheimers Dis. 2013 Jan 1;34(2):457-68.

6. Villemagne VL. Selective Tau Imaging: Der Stand der Dinge. J Nucl Med. 2017 Sep 21.

7. Johnson KA, Schultz A, Betensky RA, et al. Tau positron emission tomographic imaging in aging and early Alzheimer disease. Ann Neurol. 2016 Jan;79(1):110-9.

8. Petersen RC, Wiste HJ, Weigand SD, et al. Association of Elevated Amyloid Levels With Cognition and Biomarkers in Cognitively Normal People From the Community. JAMA Neurol. 2016 Jan;73(1):85-92.

9. Lim YY, Snyder PJ, Pietrzak RH, et al. Sensitivity of composite scores to amyloid burden in preclinical Alzheimer's disease: Introducing the Z-scores of Attention, Verbal fluency, and Episodic memory for Nondemented older adults composite score. Alzheimers Dement (Amst). 2016;2:19-26.

10. Mormino EC, Papp KV, Rentz DM, et al. Early and late change on the preclinical Alzheimer's cognitive composite in clinically normal older individuals with elevated amyloid-beta. Alzheimers Dement. 2017 Feb 28;Sep;13(9) 1004-12.

11. Donohue MC, Sperling RA, Petersen R, et al. Association Between Elevated Brain Amyloid and Subsequent Cognitive Decline Among Cognitively Normal Persons. JAMA. 2017 Jun 13;317(22):2305-16.

12. Farrell ME, Kennedy KM, Rodrigue KM, et al. Association of Longitudinal Cognitive Decline With Amyloid Burden in Middle-aged and Older Adults: Evidence for a Dose-Response Relationship. JAMA Neurol. 2017 Jul 1;74(7):830-8.

13. Scholl M, Lockhart SN, Schonhaut DR, et al. PET Imaging of Tau Deposition in the Aging Human Brain. Neuron. 2016 Mar 02;89(5):971-82. 
14. Maass A, Lockhart SN, Harrison TM, et al. Entorhinal Tau Pathology, Episodic Memory Decline, and Neurodegeneration in Aging. J Neurosci. 2018 Jan 17;38(3):53043.

15. Sperling RA, Aisen PS, Beckett LA, et al. Toward defining the preclinical stages of Alzheimer's disease: Recommendations from the National Institute on AgingAlzheimer's Association workgroups on diagnostic guidelines for Alzheimer's disease. Alzheimers Dement. 2011 May;7(3):280-92.

16. Vos SJ, Xiong C, Visser PJ, et al. Preclinical Alzheimer's disease and its outcome: a longitudinal cohort study. Lancet Neurol. 2013 Oct;12(10):957-65.

17. Soldan A, Pettigrew C, Cai Q, et al. Hypothetical Preclinical Alzheimer Disease Groups and Longitudinal Cognitive Change. JAMA Neurol. 2016 Jun 01;73(6):698-705.

18. Nelson PT, Alafuzoff I, Bigio EH, et al. Correlation of Alzheimer disease neuropathologic changes with cognitive status: a review of the literature. J Neuropathol Exp Neurol. 2012 May;71(5):362-81.

19. Mormino EC, Papp KV, Rentz DM, et al. Heterogeneity in Suspected NonAlzheimer Disease Pathophysiology Among Clinically Normal Older Individuals. JAMA Neurol. 2016 Oct 1;73(10):1185-91.

20. Mormino EC, Betensky RA, Hedden T, et al. Amyloid and APOE epsilon4 interact to influence short-term decline in preclinical Alzheimer disease. Neurology. 2014 May 20;82(20):1760-7.

21. Wang L, Benzinger TL, Su Y, et al. Evaluation of Tau Imaging in Staging Alzheimer Disease and Revealing Interactions Between beta-Amyloid and Tauopathy. JAMA Neurol. 2016 Sep 01;73(9):1070-7.

22. Lockhart SN, Scholl M, Baker SL, et al. Amyloid and tau PET demonstrate region-specific associations in normal older people. Neuroimage. 2017 Apr 15;150:191-9.

23. Villemagne VL, Dore V, Bourgeat $\mathrm{P}$, et al. Abeta-amyloid and Tau Imaging in Dementia. Semin Nucl Med. 2017 Jan;47(1):75-88.

24. Lee C, Jacobs HL, Marquie M, et al. 18F-Flortaucipir binding in choroid plexus: related to race and hippocampus signal. Journal of Alzheimer's disease. 2018;62(4).

25. Greve DN, Salat DH, Bowen SL, et al. Different partial volume correction methods lead to different conclusions: An (18)F-FDG-PET study of aging. Neuroimage. 2016 May 15;132:334-43.

26. Grothe MJ, Barthel H, Sepulcre J, et al. In vivo staging of regional amyloid deposition. Neurology. 2017 Nov 14;89(20):2031-8.

27. Gomar JJ, Conejero-Goldberg C, Davies P, Goldberg TE, Alzheimer's Disease Neuroimaging I. Anti-Correlated Cerebrospinal Fluid Biomarker Trajectories in Preclinical Alzheimer's Disease. J Alzheimers Dis. 2016;51(4):1085-97.

28. Schindler SE, Jasielec MS, Weng H, et al. Neuropsychological measures that detect early impairment and decline in preclinical Alzheimer disease. Neurobiol Aging. 2017 Aug;56:25-32. 
29. Clark L, Berman S, Norton D, et al. Age-accelerated cognitive decline in asymptomatic adults with CSF $\beta$-amyloid. Neurology. 2018;[Epub ahead of print]. 30. Jin M, Shepardson N, Yang T, Chen G, Walsh D, Selkoe DJ. Soluble amyloid beta-protein dimers isolated from Alzheimer cortex directly induce Tau hyperphosphorylation and neuritic degeneration. Proc Natl Acad Sci U S A. 2011 Apr 05;108(14):5819-24.

31. Mormino EC, Betensky RA, Hedden T, et al. Synergistic Effect of beta-Amyloid and Neurodegeneration on Cognitive Decline in Clinically Normal Individuals. JAMA Neurology. 2014 Sep 15;epub 2014/09/16.

32. Burnham SC, Bourgeat P, Dore V, et al. Clinical and cognitive trajectories in cognitively healthy elderly individuals with suspected non-Alzheimer's disease pathophysiology (SNAP) or Alzheimer's disease pathology: a longitudinal study. Lancet Neurol. 2016 Sep;15(10):1044-53.

33. Machulda MM, Hagen CE, Wiste HJ, et al. [Formula: see text]Practice effects and longitudinal cognitive change in clinically normal older adults differ by Alzheimer imaging biomarker status. Clin Neuropsychol. 2017 Jan;31(1):99-117.

34. Hanseeuw BJ, Betensky RA, Schultz AP, et al. Fluorodeoxyglucose metabolism associated with tau-amyloid interaction predicts memory decline. Ann Neurol. 2017 Apr;81(4):583-96.

35. Crary JF, Trojanowski JQ, Schneider JA, et al. Primary age-related tauopathy (PART): a common pathology associated with human aging. Acta Neuropathol. 2014 Dec;128(6):755-66.

36. Josephs KA, Murray ME, Tosakulwong N, et al. Tau aggregation influences cognition and hippocampal atrophy in the absence of beta-amyloid: a clinico-imagingpathological study of primary age-related tauopathy (PART). Acta Neuropathol. 2017 May;133(5):705-15.

37. Vemuri P, Lowe VJ, Knopman DS, et al. Tau-PET uptake: Regional variation in average SUVR and impact of amyloid deposition. Alzheimers Dement (Amst). 2017;6:21-30.

38. Lowe VJ, Wiste HJ, Senjem ML, et al. Widespread brain tau and its association with ageing, Braak stage and Alzheimer's dementia. Brain. 2018 Jan 1;141(1):271-87. 39. Sperling R, Mormino E, Johnson K. The evolution of preclinical Alzheimer's disease: implications for prevention trials. Neuron. 2014 Nov 5;84(3):608-22.

40. Braak H, Braak E. Neuropathological stageing of Alzheimer-related changes. Acta Neuropathol. 1991;82(4):239-59.

41. Marquie M, Normandin MD, Vanderburg CR, et al. Validating novel tau positron emission tomography tracer [F-18]-AV-1451 (T807) on postmortem brain tissue. Ann Neurol. 2015 Nov;78(5):787-800. 
42. Reitz C, Honig L, Vonsattel JP, Tang MX, Mayeux R. Memory performance is related to amyloid and tau pathology in the hippocampus. J Neurol Neurosurg Psychiatry. 2009 Jul;80(7):715-21.

43. Braak H, Braak E. Frequency of stages of Alzheimer-related lesions in different age categories. Neurobiol Aging. 1997 Jul-Aug;18(4):351-7.

44. Teichmann M, Epelbaum S, Samri D, et al. Free and Cued Selective Reminding Test - accuracy for the differential diagnosis of Alzheimer's and neurodegenerative diseases: a large-scale biomarker-characterized monocenter cohort study (ClinAD). Alzheimers Dement. 2017 Feb 21.

45. Rentz DM, Mormino EC, Papp KV, Betensky RA, Sperling RA, Johnson KA. Cognitive resilience in clinical and preclinical Alzheimer's disease: the Association of Amyloid and Tau Burden on cognitive performance. Brain Imaging Behav. 2017 Apr;11(2):383-90.

46. Dubois B, Hampel H, Feldman HH, et al. Preclinical Alzheimer's disease: Definition, natural history, and diagnostic criteria. Alzheimers Dement. 2016 Mar;12(3):292-323.

47. Hyman BT. Amyloid-dependent and amyloid-independent stages of Alzheimer disease. Arch Neurol. 2011 Aug;68(8):1062-4.

48. Sperling RA, Rentz DM, Johnson KA, et al. The A4 study: stopping AD before symptoms begin? Sci Transl Med. 2014 Mar 19;6(228):228fs13.

49. Bateman RJ, Aisen PS, De Strooper B, et al. Autosomal-dominant Alzheimer's disease: a review and proposal for the prevention of Alzheimer's disease. Alzheimers Res Ther. 2011 Jan 6;3(1).

50. Langbaum JB, Fleisher AS, Chen K, et al. Ushering in the study and treatment of preclinical Alzheimer disease. Nat Rev Neurol. 2013 Jul;9(7):371-81. 


\section{Figure Legends}

Figure 1: Vertex-wise map of Flortaucipir tau (FTP) PET (threshold set at $\mathrm{p}<0.001$ ) demonstrating regions with higher tau associated with higher levels of cortical amyloid (PiB), controlling for age. Left hemisphere is shown on the left side of the figure. The color bar represents T values, with warmer colors reflecting vertices with stronger associations between $\mathrm{A} \beta$ and tau.

Figure 2: Plots of tau vs. Memory and Executive (EF) Slope, with each plotted variable residualized by age, sex, education, APOE4, and time of tau scan. LOESS lines are fit in each plot. Participants are divided by dichotomous $A \beta$ group for illustration purposes, as $\mathrm{A} \beta$ was considered as a continuous variable in the statistical models, for: $(\mathrm{A}, \mathrm{D})$ entorhinal cortex (EC), (B, E) hippocampus (Hip), and (C, F) Inferior Temporal cortex tau. No relationship between tau and memory was observed among the A $\beta$ - group (black circles) in any ROI, however, the range of tau is more limited in the $A \beta$ - group. The $A \beta+$ (red diamonds) demonstrate a negative association between tau and memory slope (top row) across all three regions. The three participants who progressed to a clinical diagnosis of MCI within one year of their tau scan date (all were $\mathrm{A} \beta+$ ), are indicated in the green triangles. In addition, there were two participants who demonstrated substantial memory decline from their baseline but did not yet meet MCI criteria. The interaction 
analyses remained significant with these participants excluded. Neither A $\beta$ group demonstrated clear evidence of a relationship between tau and EF.

Figure 3: Vertex-wise maps of 11-C-Pittsburg Compound B (PiB) and 18-F-Flortaucipir (FTP) tau PET imaging demonstrating relationship with memory slopes across all participants, thresholded at $\mathrm{p}<0.001$. Left hemisphere is shown on the left side of the figure. Color bar represents $\mathrm{T}$ values with warmer colors connoting vertices with stronger relationship between higher FTP SUVR and memory decline. A) Map of PiB and memory change demonstrating multiple cortical regions, including orbital and lateral prefrontal, precuneus and posterior cingulate, and lateral parietal cortices, where higher $\mathrm{A} \beta$ burden is associated with decreasing memory performance: $\mathrm{B}$ ) Map of FTP and memory change showing multiple regions in medial and inferior temporal cortices in which higher levels of FTP binding are associated with more rapid prospective decline in memory. C) Map of PiB interacting with inferior temporal tau ROI reveals areas of precuneus, posterior cingulate, lateral prefrontal, and lateral parietal cortices that demonstrate significant association with memory decline. D) Map of FTP interacting with cortical A $\beta$ ROI demonstrates regions in medial and lateral temporal cortices, as well as limited areas of cingulate and prefrontal cortices associated with memory decline. Notably these tau regions interacting with $\mathrm{A} \beta$ (in $\mathrm{D}$ ) show a stronger relationship than the tau regions alone (in B).

p. 33

This article is protected by copyright. All rights reserved. 
Figure 4: Demonstration of the three-way interaction between continuous $\mathrm{A} \beta \mathrm{x} \mathrm{EC}$ tau $\mathrm{x}$ Time predicting memory. The top row shows the effect of Amyloid on memory change at varying levels of EC tau. For reference, the distribution of EC tau values in the sample is shown to the left. The effect of Amyloid at different levels of tau is plotted on the right (for instance the green vertical line at left represents EC tau SUVR=1.0 and corresponds to the green point at right showing the multiplicative effect of Amyloid burden is 0.0 when EC tau SUVR is 1.0). This plot demonstrates that the effect of Amyloid increases at higher levels of EC tau, with a negative relationship between Amyloid and memory change emerging around values of 1.1 SUVR in EC tau (blue dot at right). Likewise, the bottom row shows the effect of EC tau at varying levels of global Amyloid (for instance the green point reflects the effect of EC tau when Amyloid DVR is 1.05). For reference, the distribution of global Amyloid DVR values in the sample is shown to the left. This plot shows that the negative effect of EC tau on memory emerges at higher levels of Amyloid (around DVR values of 1.4). This multiplicative effect of A $\beta$ and tau was consistent across all examined tau regions, as indicated by the significant interaction term across all three models. 
Tables

TABLE 1. Demographic and Cognitive Test Performance

\begin{tabular}{|c|c|c|c|}
\hline & All Participants & $A \beta-$ & $\mathrm{A} \beta+$ \\
\hline $\mathrm{N}$ & 137 & 97 & 40 \\
\hline \multirow[t]{2}{*}{ Age at Tau PET scan\# } & $76.32(6.22)$ & $75.54(6.41)$ & $78.20(5.34)$ \\
\hline & Range $=65-90$ & Range $=65-90$ & Range $=68-88$ \\
\hline Female Sex & 79 & 55 & 24 \\
\hline Years of Education & 15.85 (2.95) & 15.75 (3.07) & $16.10(2.67)$ \\
\hline $\begin{array}{l}\text { APOE \&4 non- } \\
\text { carrier/APOE } \varepsilon 4 \\
\text { carrier* }^{*}\end{array}$ & $94 / 43$ & $81 / 16$ & $13 / 27$ \\
\hline $\begin{array}{l}\text { Total Prospective } \\
\text { Follow Up Time } \\
\text { (years) }\end{array}$ & $2.05(0.74)$ & $2.09(0.72)$ & $1.94(0.76)$ \\
\hline $\begin{array}{l}\text { Total number of } \\
\text { cognitive test visits }\end{array}$ & $2.8(1.1)$ & $2.8(1.2)$ & $2.8(1.1)$ \\
\hline MMSE & $29.18(1.04)$ & $29.29(0.98)$ & $28.92(1.14)$ \\
\hline $\begin{array}{l}\text { Logical Memory } \\
\text { Delayed Recall WMS } \\
\text { IIa** }^{*}\end{array}$ & $15.75(3.92)$ & $16.30(3.81)$ & 14.43 (3.93) \\
\hline
\end{tabular}

Table 1: Demographics and cognitive test performance (mean [S.D.]) at clinic visit corresponding to year of Tau and A $\beta$ PET imaging for the entire sample and classified by dichotomous $A \beta$ status. Mean and standard deviation are listed for continuous variables. 
* Age difference $\mathrm{p}<0.05$ between $\mathrm{A} \beta$ - and $\mathrm{A} \beta$ + groups. ${ }^{\text {\#Total prospective follow up }}$ time was defined using the neuropsychological testing session within one year of the Tau PET as the "baseline" visit and comparing that date to the date of the final subsequent neuropsychological testing session. Likewise, the tabulated MMSE and Logical Memory values reflect scores from the neuropsychological testing session within one year of the date of the tau scan. ${ }^{* *}$ Logical Memory performance difference $\mathrm{p}<0.05$ between $A \beta$ - and $\mathrm{A} \beta+$ groups. 
TABLE 2. A $\beta$ and tau modeled as simultaneous predictors of memory and executive function change

\begin{tabular}{|c|c|c|c|}
\hline & EC Tau & Hip Tau & IT Tau \\
\hline \multirow{4}{*}{ Memory } & Age $x$ Time & Age $x$ Time & Age $x$ Time \\
\hline & $\begin{array}{c}-0.008(0.005) \\
p=0.12\end{array}$ & $\begin{array}{c}-0.007(0.005) \\
\mathrm{p}=0.20\end{array}$ & $\begin{array}{c}-0.009(0.005) \\
\mathrm{p}=0.095\end{array}$ \\
\hline & A $\beta$ cont $x$ EC tau $x$ Time & $\begin{array}{c}\text { A } \beta \text { cont } x \text { Hip tau } x \\
\text { Time }\end{array}$ & A $\beta$ cont $x$ IT tau $x$ Time \\
\hline & $\begin{array}{c}-4.261(1.057) \\
\mathrm{p}<0.001\end{array}$ & $\begin{array}{c}-7.044(1.520) \\
\mathrm{p}<0.001\end{array}$ & $\begin{array}{c}-5.160(1.360) \\
\mathrm{p}<0.001\end{array}$ \\
\hline \multirow{4}{*}{$\begin{array}{l}\text { Executive } \\
\text { Function }\end{array}$} & Age $x$ Time & Age $x$ Time & Age $x$ Time \\
\hline & $\begin{array}{c}-0.009(0.003) \\
p=0.0019\end{array}$ & $\begin{array}{c}-0.009(0.003) \\
\mathrm{p}<0.001\end{array}$ & $\begin{array}{c}-0.009(0.0028) \\
\mathrm{p}=0.002\end{array}$ \\
\hline & $A \beta$ cont $x E C$ tau $x$ Time & $\begin{array}{c}\text { A } \beta \text { cont } x \text { Hip tau } x \\
\text { Time } \\
\end{array}$ & A $\beta$ cont $x$ IT tau $x$ Time \\
\hline & $\begin{array}{c}0.065(0.5449) \\
\mathrm{p}=0.91\end{array}$ & $\begin{array}{c}0.623(0.776) \\
\mathrm{p}=0.42\end{array}$ & $\begin{array}{c}-0.085(0.7094) \\
p=0.91\end{array}$ \\
\hline
\end{tabular}

Table 2. Linear mixed models with $A \beta$ and tau as simultaneous predictors of memory and executive function change, repeated for each tau region. Unstandardized betas and standard errors are listed. All models include the two-way interactions between $\mathrm{A} \beta \mathrm{x}$ Time and tau $\mathrm{x}$ Time, as well as covariates for APOE4, education, sex, and time of tau scan, as well as their interactions with time. Significant effects are highlighted in gray $(\mathrm{p}<0.05)$. 
TABLE 3. Relationship of Tau Regions to Memory Decline by Dichotomous A $\beta$ group.

\begin{tabular}{|c|c|c|}
\hline & $\mathrm{A} \beta-$ & $\mathrm{A} \beta+$ \\
\hline EC Tau $x$ Time & $0.725 \pm 0.472$ & $-1.656 \pm 0.657$ \\
& $\mathrm{p}=0.13$ & $\mathrm{p}=0.012$ \\
\hline Hip Tau x Time & $0.013 \pm 0.513$ & $-2.310 \pm 0.649$ \\
& $\mathrm{p}=0.98$ & $\mathrm{p}<0.001$ \\
\hline IT Tau x Time & $0.676 \pm 0.573$ & $-1.643 \pm 0.486$ \\
& $\mathrm{p}=0.24$ & $\mathrm{p}<0.001$ \\
\hline
\end{tabular}

Table 3. Linear mixed models were repeated with $\mathrm{A} \beta$ treated as a dichotomous variable, and the effect of tau on memory change (Regional tau $x$ Time) was estimated in $A \beta$ and $A \beta+$ groups from these models for Entorhinal cortex (EC), Hippocampus (Hip) and Inferior Temporal (IT) cortex. Unstandardized betas and standard errors are listed. All models additionally include covariates for APOE4, education, sex, and time of tau scan, as well as their interactions with time. A consistent pattern is observed, such that elevated regional tau is associated with greater memory decline over time across all regions only in the $\mathrm{A} \beta+$ group. Significant effects are highlighted in gray $(\mathrm{p}<0.05)$. 


\section{SUPPLEMENTAL TABLE 1: Equations for Linear Mixed Models}

LMM with $\mathrm{A} \beta$

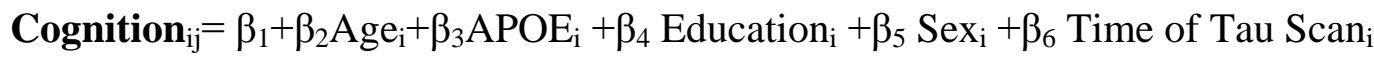

$+\boldsymbol{\beta}_{7} \mathbf{A} \boldsymbol{\beta}_{\mathbf{i}}+\beta_{8}$ Time $_{i j}+\left[\beta_{9}\right.$ Age $_{i} \times$ Time $\left._{i j}\right]+\left[\beta_{10}\right.$ APOE $_{\mathrm{i}} \mathrm{x}$ Time $\left._{\mathrm{ij}}\right]+\left[\beta_{11}\right.$ Education $_{\mathrm{i}} \mathrm{x}$ Time $\left._{\mathrm{ij}}\right]$

$+\left[\beta_{12} \operatorname{Sex}_{\mathrm{i}} \times\right.$ Time $\left._{\mathrm{ij}}\right]+\left[\boldsymbol{\beta}_{13}\right.$Time of Tau Scan $_{\mathrm{i}} \mathrm{x}$ Time $\left._{\mathrm{ij}}\right]+\left[\boldsymbol{\beta}_{\mathbf{1 4}} \mathbf{A} \boldsymbol{\beta}_{\mathbf{i}} \mathbf{x} \mathbf{T i m e}_{\mathbf{i j}}\right]+\mathrm{b}_{1 \mathrm{i}}$

LMM with regional Tau

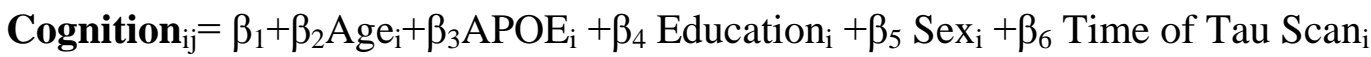
$+\boldsymbol{\beta}_{7}$ Regional Tau $_{\mathbf{i}}+\beta_{8}$ Time $_{\mathrm{ij}}+\left[\beta_{9}\right.$ Age $_{\mathrm{i}} \mathrm{x}$ Time $\left._{\mathrm{ij}}\right]+\left[\beta_{10}\right.$ APOE $_{\mathrm{i}} \mathrm{x}$ Time $\left._{\mathrm{ij}}\right]+\left[\beta_{11}\right.$ Education $_{\mathrm{i}}$

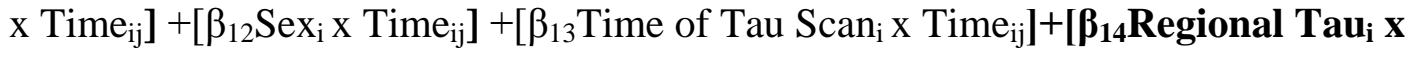
Time $\left._{i j}\right]+b_{1 i}$

Cognition $_{\mathrm{ij}}=$ Memory or executive function composite score at each testing session Age $_{i}=$ Age at Tau scan

$\mathrm{APOE}_{\mathrm{i}}=\mathrm{APOE} 4$ carrier status (carrier versus non-carrier)

Education $_{\mathrm{i}}=$ Education (in years)

Sex $_{\mathrm{i}}=$ Male versus Female

Time of Tau $\operatorname{Scan}_{\mathrm{i}}=$ Time of Tau scan relative to study baseline. Dichotomized into 2 levels (either early in the study, between the $1^{\text {st }}$ and $3^{\text {rd }}$ annual visit; or late in the study, during the $4^{\text {th }}$ visit or later).

This article is protected by copyright. All rights reserved. 
Time $_{\mathrm{ij}}=$ Time relative to the testing session up to one year before the tau PET scan (all testing sessions more than one year before Tau PET were discarded to enable examination of prospective change in cognition).

$\mathrm{A} \beta_{\mathrm{i}}=$ Continuous levels of $\mathrm{A} \beta$ (from PiB scans closest to tau PET scan)

Regional tau $\mathrm{i}_{\mathrm{i}} \mathrm{EC} \mathrm{Tau}$, HV Tau $\mathrm{T}_{\mathrm{i}}$, or IT Tau $\mathrm{Talues}_{\mathrm{i}}$

$\mathrm{b}_{1 \mathrm{i}}=$ random intercept for each subject 


\section{University Library}

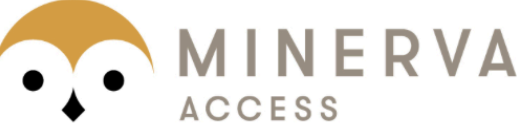

A gateway to Melbourne's research publications

Minerva Access is the Institutional Repository of The University of Melbourne

Author/s:

Sperling, RA;Mormino, EC;Schultz, AP;Betensky, RA;Papp, KV;Amariglio, RE;Hanseeuw, BJ;Buckley, R;Chhatwal, J;Hedden, T;Marshall, GA;Quiroz, YT;Donovan, NJ;Jackson, J;Gatchel, JR;Rabin, JS;Jacobs, H;Yang, H-S;Properzi, M;Kirn, DR;Rentz, DM;Johnson, KA

Title:

The impact of amyloid-beta and tau on prospective cognitive decline in older individuals

Date:

2019-02-01

Citation:

Sperling, R. A., Mormino, E. C., Schultz, A. P., Betensky, R. A., Papp, K. V., Amariglio, R. E., Hanseeuw, B. J., Buckley, R., Chhatwal, J., Hedden, T., Marshall, G. A., Quiroz, Y. T., Donovan, N. J., Jackson, J., Gatchel, J. R., Rabin, J. S., Jacobs, H., Yang, H. -S., Properzi, M. ,... Johnson, K. A. (2019). The impact of amyloid-beta and tau on prospective cognitive decline in older individuals. ANNALS OF NEUROLOGY, 85 (2), pp.181-193. https://doi.org/10.1002/ana.25395.

Persistent Link:

http://hdl.handle.net/11343/284965 